\title{
Finite Groups with Some SE-Supplemented Subgroups
}

\author{
Guo Zhong, ${ }^{1}$ Liying Yang, ${ }^{1}$ Huaquan $\mathrm{Wei}^{2}{ }^{2}$ Xuanlong $\mathrm{Ma}^{3}$ and Jiayi Xia ${ }^{1}$ \\ ${ }^{1}$ School of Mathematical Sciences, Guangxi Teachers Education University, Nanning, Guangxi 530023, China \\ ${ }^{2}$ College of Mathematics and Information Science, Guangxi University, Nanning, Guangxi 530004, China \\ ${ }^{3}$ School of Mathematical Sciences, Beijing Normal University, Beijing 100875, China
}

Correspondence should be addressed to Guo Zhong; zhg102003@163.com

Received 30 March 2013; Accepted 29 May 2013

Academic Editor: Antonio M. Cegarra

Copyright (C) 2013 Guo Zhong et al. This is an open access article distributed under the Creative Commons Attribution License, which permits unrestricted use, distribution, and reproduction in any medium, provided the original work is properly cited.

Let $H$ be a subgroup of a finite group $G, p$ a prime dividing the order of $G$, and $P$ a Sylow $p$-subgroup of $G$ for prime $p$. We say that $H$ is $S E$-supplemented in $G$ if there is a subgroup $K$ of $G$ such that $G=H K$ and $H \cap K \leq H_{\text {seG }}$, where $H_{\text {seG }}$ denotes the subgroup of $H$ generated by all those subgroups of $H$ which are $S$-quasinormally embedded in $G$. In this paper, we characterize $p$-nilpotency and supersolvability of $G$ under the assumption that all maximal subgroups of $P$ are $S E$-supplemented in $N_{G}(P)$.

\section{Introduction}

Throughout only finite groups are considered. Let $\pi(G)$ stand for the set of all prime divisors of the order of a group $G$. $\mathcal{U}$ denotes the class of all supersolvable groups. $H$ char $G$ means $H$ is a characteristic subgroup of $G$. We use conventional notions and notation, as in Robinson [1].

Let $\mathscr{F}$ be a class of groups. $\mathscr{F}$ is called a formation provided that (1) if $G \in \mathscr{F}$ and $H \triangleleft G$, then $G / H \in \mathscr{F}$ and (2) if $G / M$ and $G / N$ are in $\mathscr{F}$, then $G / M \cap N$ is in $\mathscr{F}$ for all normal subgroups $M, N$ of $G$. A formation $\mathscr{F}$ is said to be saturated if $G / \Phi(G) \in \mathscr{F}$ implies that $G \in \mathscr{F}$.

Two subgroups $H$ and $K$ of a group $G$ are said to be permutable if $H K=K H . H$ is said to be $S$-quasinormal in $G$ if $H$ permutes with every Sylow subgroup of $G$, that is, $H P=$ $P H$ for any Sylow subgroup $P$ of $G$. This concept was introduced by Kegel in [2] and has been studied widely by many authors, such as $[3,4]$. An interesting question in the theory of finite groups is to determine the influence of the embedding properties of members of some distinguished families of subgroups of a group on the structure of the group. Recently, Ballester-Bolinches and Pedraza-Aguilera [5] generalized $S$ quasinormal subgroups to $S$-quasinormally embedded subgroups. $H$ is said to be $S$-quasinormally embedded in $G$ provided every Sylow subgroup of $H$ is a Sylow subgroup of some $S$-quasinormal subgroup of $G$. By applying this concept, Ballester-Bolinches and Pedraza-Aguilera got new criteria for supersolvability of groups.

A subgroup $H$ of a group $G$ is called to be complemented in $G$ if $G$ has a subgroup $K$ such that $G=H K$ and $H \cap K=1$. $H$ is called to be supplemented in $G$ if there exists a subgroup $T$ of $G$ such that $G=H T$. Obviously, a complemented subgroup is a special supplemented subgroup. In recent years, it has been of interest to use supplementation properties of subgroups to characterize properties of a group. For example, the definition of a weakly $S$-supplemented subgroup introduced by Skiba in [6]. $H$ is said to be weakly $S E$ supplemented in $G$ if $G$ has a subgroup $U$ such that $H U=G$ and $H \cap U \leq H_{s G}$, where $H_{s G}$ is the largest $S$-quasinormal subgroup of $G$ contained in $H$. Using this concept, many meaningful results have been obtained, such as [7-9]. More recently, the concept of SE-supplemented subgroups was introduced as follows.

Definition 1 (see [10, Definition 1.2]). Let $H$ be a subgroup of $G$. We say that $H$ is $S E$-supplemented in $G$ if there exists some subgroup $T$ of $G$ such that $H T=G$ and $H \cap T \leq H_{s e G}$, where $H_{s e G}$ denotes the subgroup generated by all the subgroups of $H$ which are $S$-quasinormally embedded in $G$. We call $H_{s e G}$ the $S E$-core of $H$ in $G$. 
Clearly, normal subgroups, $S$-quasinormal subgroups, $S$ quasinormally embedded subgroups, and weakly $S$-supplemented subgroups are all SE-supplemented subgroups. But the converse does not hold in general (see [11]). Based on the observation of the above concepts, we note that supplementation of some families of subgroups of a group has a strong influence on its structure.

On the other hand, the normalizer of a Sylow subgroup of a group takes an important role in studying the structure of a group. Let $P$ be a Sylow subgroup of a group $G$; it is natural to consider the structure of $G$ if some properties of the normalizer $N_{G}(P)$ of $P$ are known. A classical result in this orientation is attributed to Burnside's Theorem [1, Theorem 10.1.8]. Later, Hall [12] extended it as follows: if each $p^{\prime}$ element of $N_{G}(P)$ does commute with all elements of $P$ and if also the class size of $P$ is less than $p$, then $G$ is $p$-nilpotent. In short, it is of significance to research into the structure of finite groups from properties of the normalizer of a Sylow subgroup of a group. In this paper, under the assumption that all maximal subgroups of a Sylow subgroup $P$ are $S E$ supplemented in $N_{G}(P)$, we give some new characterizations on the structure of $G$.

\section{Preliminaries}

For the sake of convenience, we first cite some known results in the literature which will be useful in the following.

Lemma 2 (see [13, Lemma 2.1]). Let $H$ and $K$ be subgroups of a group $G$.

(1) If $H$ is $S$-quasinormal in $G$ and $H \leq M \leq G$, then $H$ is $S$-quasinormal in $M$.

(2) Let $N \triangleleft G$ and $H$ be $S$-quasinormal in $G$. Then $H N$ is $S$-quasinormal in $G$ and $H N / N$ is $S$-quasinormal in G/N.

(3) If $H$ is $S$-quasinormal in $G$, then $H$ is subnormal in $G$.

(4) If both $H$ and $K$ are $S$-quasinormal subgroups of $G$, then both $H \cap K$ and $\langle H, K\rangle$ are $S$-quasinormal subgroups of $G$.

Lemma 3 (see [10, Lemma 2.6]). Suppose that $N$ is a normal subgroup of $G$ and $H \leq K \leq G$. Then

(1) $H_{s e G} \unlhd H$;

(2) $H_{s e G} \leq H_{s e K}$;

(3) $H_{s e G} N / N \leq(H N / N)_{s e(G / N)}$;

(4) If $(|N|,|H|)=1$, then $H_{s e G} N / N=(H N / N)_{s e(G / N)}$.

Lemma 4 (see [10, Lemma 2.7]). Let $H$ be a subgroup of $a$ group $G$.

(1) If $H$ is $S E$-supplemented in $G$ and $H \leq M \leq G$, then $H$ is SE-supplemented in $M$.

(2) Let $N \triangleleft G$ and $N \leq H$. If $H$ is $S E$-supplemented in $G$, then $H / N$ is $S E$-supplemented in $G / N$.

(3) Let $\pi$ be a set of primes, $H$ a $\pi$-subgroup of $G$, and $N$ a normal $\pi^{\prime}$-subgroup of $G$. If $H$ is SE-supplemented in $G$, then $H N / N$ is SE-supplemented in $G / N$.
Lemma 5 (see [14]). Let $G$ be a group.

(1) If $P$ is an $S$-quasinormal p-subgroup of $G$ for some prime $p$, then $N_{G}(P) \geq O^{p}(G)$.

(2) Suppose that $H$ is a p-subgroup of $G$ contained in $\mathrm{O}_{p}(G)$. If $H$ is $S$-quasinormally embedded in $G$, then $H$ is $S$-quasinormal in $G$.

Lemma 6. Let $H$ be a subgroup of a group $G$. If $H$ is $S E-$ supplemented in $G$ and $H \leq O_{p}(G)$, then $H$ is weakly $S E$ supplemented in $G$.

Proof. In fact, $H_{s e G}=\left\langle H_{1}, H_{2}, \ldots, H_{s}\right\rangle$, where $H_{i}(i=1,2$, $\ldots, s)$ is $S$-quasinormally embedded in $G$. Since $H \leq O_{p}(G)$, we have that $H_{i}$ is $S$-quasinormal in $G$ by Lemma 5(2). Thus, $H_{s e G} \leq H_{s G}$, where $H_{s G}$ is the largest $S$-quasinormal subgroup of $G$ contained in $H$. Consequently, $H$ is weakly $S E$ supplemented in $G$.

Lemma 7 (see [15, Lemma 2.1]). Let $G$ be a group. If $A$ is subnormal in $G$ and $A$ is a $\pi$-subgroup of $G$, then $A \leq O_{\pi}(G)$.

From Lemma 6, the following Lemma is a direct corollary of Lemma 3.1 in [7].

Lemma 8. Suppose $G=P Q$, where $P$ is a normal Sylow $p$ subgroup and $Q$ a Sylow q-subgroup of $G$. If $(|G|, p-1)=1$ and if also every maximal subgroup of $P$ is SE-supplemented in $G$, then $G$ is p-nilpotent.

\section{Main Results}

Theorem 9. Let $G$ be a group and assume $p$ is a prime dividing the order of $G$ with $(|G|, p-1)=1$. If there exists a Sylow $p$ subgroup $P$ of $G$ such that every maximal subgroup of $P$ is $S E$ supplemented in $N_{G}(P)$ and if also $P^{\prime}$ is $S$-quasinormal in $G$, then $G$ is p-nilpotent.

Proof. Suppose that the result is not true and let $G$ be a counterexample of minimal order. We will derive a contradiction in several steps.

(1) $O_{p}(G) \geq P^{\prime} \neq 1$. Let $Q \in \operatorname{Syl}_{q}\left(N_{G}(P)\right)$, where $q$ is a prime number dividing $\left|N_{G}(P)\right|$ and different from $p$. We can see that all maximal subgroups of $P$ are $S E$-supplemented in $P Q$ by Lemma 4 . Then PQ meets the hypothesis of Lemma 8 . It follows that $P Q$ is $p$-nilpotent and thus $Q \leq C_{G}(P)$. We know that all $p^{\prime}$-elements of $N_{G}(P)$ are contained in $C_{G}(P)$. If $P$ is abelian, then $N_{G}(P)=C_{G}(P)$, which yields that $G$ is $p$ nilpotent from Burnside's theorem [1, Theorem 10.1.8], which is a contradiction. Thus we may assume that $P^{\prime} \neq 1$. Since $P^{\prime}$ is $S$-quasinormal in $G$, thus $P^{\prime}$ is subnormal in $G$ by Lemma 2 . It follows from Lemma 7 that $1 \neq P^{\prime} \leq O_{p}(G)$.

(2) For any Normal Subgroup $N$ of $G$ Contained in $P, G / N$ is $p$-Nilpotent and $G$ is Solvable. It is clear that $(|G / N|, p-1)=1$. For any maximal subgroup $P_{1} / N$ of $P / N, P_{1}$ is a maximal subgroup of $P$. From this hypothesis, $P_{1}$ is $S E$-supplemented in $N_{G}(P)$ and $P^{\prime}$ is $S$-quasinormal in $G$. It follows by Lemma 4 
that $P_{1} / N$ is $S E$-supplemented in $N_{G}(P) / N=N_{G} / N(P / N)$, and $(P / N)^{\prime}=P^{\prime} N / N$ is $S$-quasinormal in $G / N$ by Lemma 2 . As a result, $G / N$ meets the hypothesis of the theorem. The choice of $G$ yields that $G / N$ is $p$-nilpotent. Let $M / N$ be a normal $p$-complement of $G / N$. If $p=2$, then $M / N$ is a group of odd order. It follows from the Feit-Thompson theorem which asserts that every group of odd order is solvable, so is $M$. We note that $G / M$ is a 2-group, and so $G$ is solvable. If $p \neq 2$, then $G$ is a group of odd order by $(|G|, p-1)=1$. Similarly, it deduces that $G$ is solvable, too.

(3) $|G|=p^{a} q^{b}$ for Some Prime $q \neq p$. Since $G$ is solvable, there exists a Sylow system $P_{1}=\left\{P, P_{2}, \ldots, P_{s}\right\}$ of $G$ with $G_{i}=P P_{i}$ for $2 \leq i \leq s$. By Lemmas 2 and 4 , the hypothesis still holds for every $G_{i}$. If $|\pi(G)|>2$, then $G_{i}<G$ and thus $G_{i}$ is $p$-nilpotent by the choice of $G$, whence $P_{i} \unlhd G_{i}$, thereby meaning that $P$ normalizes $P_{i}$ for each $2 \leq i \leq s$. Therefore $G$ is $p$-nilpotent and $K=P_{2} P_{3} \cdots P_{s}$ is a normal $p$-complement of $G$, which is a contradiction. Now, we may assume that $|G|=p^{a} q^{b}$.

(4) The Final Contradiction. Let $N$ be a minimal normal subgroup of $G$. Because $P^{\prime}$ is $S$-quasinormal in $G$ and $N \unlhd G$, $P^{\prime} N / N$ is $S$-quasinormal in $G / N$ by Lemma 2. Now we consider the quotient group $G / N$. If $N$ is a q-group, then $P N / N \in \operatorname{Syl}_{p}(G / N)$, and for any maximal subgroup $M / N$ of $P N / N$, we have $M=P_{1} N$, where $P_{1}$ is a maximal subgroup of $P$. From this hypothesis, $P_{1}$ is $S E$-supplemented in $N_{G}(P)$. Then there is a subgroup $T$ of $G$ such that $N_{G}(P)=P_{1} T$ and $P_{1} \cap T \leq\left(P_{1}\right)_{\text {seN }_{G}(P)}$. Since $P \in \operatorname{Syl}_{p}(G)$, we know that

$$
N_{G / N}\left(\frac{P N}{N}\right)=\frac{N_{G}(P) N}{N}=\left(\frac{P_{1} N}{N}\right)\left(\frac{T N}{N}\right) .
$$

As $\left(\left|P_{1}\right|,|N|\right)=1$,

$$
\begin{aligned}
\left|P_{1} \cap T N\right| & =\frac{\left(\left|P_{1}\right| \cdot|T N|_{p}\right)}{\left|P_{1} T N\right|_{p}}=\frac{\left(\left|P_{1}\right| \cdot|T|_{p}\right)}{\left|N_{G}(P) N\right|_{p}} \\
& =\frac{\left(\left|P_{1}\right| \cdot|T|_{p}\right)}{\left|N_{G}(P)\right|_{p}}=\left|P_{1} \cap T\right| .
\end{aligned}
$$

This means that $P_{1} \cap T N=P_{1} \cap T$, and thus we have

$$
\begin{aligned}
\left(\frac{P_{1} N}{N}\right) \cap\left(\frac{T N}{N}\right) & =\frac{\left(P_{1} N \cap T N\right)}{N}=\frac{\left(P_{1} \cap T N\right) N}{N} \\
& =\frac{\left(P_{1} \cap T\right) N}{N} \leq \frac{\left(P_{1}\right)_{\operatorname{seN}_{G}(P)} N}{N} .
\end{aligned}
$$

By Lemma 3, it follows that $\left(P_{1}\right)_{\operatorname{seN}_{G}(P)} N / N \leq\left(P_{1} N /\right.$ $N)_{s e\left(N_{G / N}(P N / N)\right)}$ and thus $G / N$ meets the hypothesis of our theorem. The choice of $G$ implies that $G / N$ is $p$-nilpotent, and so is $G$, contradicting the fact that $G$ is a counterexample of minimal order. Consequently, we may assume that $N$ is a $p$-group and thus $N \leq P$. It follows by (2) that $G / N$ is $p$ nilpotent. Hence we may assume that $N$ is the unique minimal normal subgroup of $G$ and $N \notin \Phi(G)$. These mean that $\Phi(G)=1$ and $O_{p}(G)=F(G)=N$.
As $P^{\prime}$ is $S$-quasinormal in $G$, we know that $N_{G}\left(P^{\prime}\right) \geq$ $O^{p}(G)$ by Lemma 5. Since $P$ normalizes $P^{\prime}$, we get that $P^{\prime} \unlhd$ $G$, then $P^{\prime}=O_{p}(G)=N$ since $N$ is the unique minimal normal subgroup of $G$. It follows from (2) that $G / O_{p}(G)$ is p-nilpotent; thus $O_{p}(G) Q \unlhd G$, where $Q \in \operatorname{Syl}_{q}(G)$. Since $O_{p}(G) Q \cap P=O_{p}(G)=P^{\prime} \leq \Phi(P), O_{p}(G) Q$ is $p$-nilpotent by Tate's Theorem [16, Theorem 4.4.7]. Thus $Q$ char $O_{p}(G) Q \unlhd G$ which yields that $Q \unlhd G$; that is, $G$ is $p$ nilpotent, a contradiction. The final contradiction completes the proof.

Remark 10. In Theorem 9, the condition that $P^{\prime}$ is $S$-quasinormal in $G$ cannot be removed.

Example 11. Let $G=\operatorname{PSL}_{2}(q)$, where $q>1$ and $q \equiv \pm 1(\bmod$ 8). Let $P$ be a Sylow 2 -subgroup of G. By [16, II, Theorem 8.27], we know that $P$ is self-normalizing in $\operatorname{PSL}_{2}(q)$. Clearly, every maximal subgroup of $P$ is normal in $N_{G}(P)=P$, and thus they are all $S E$-supplemented in $N_{G}(P)$. However, $G$ is not 2-nilpotent.

Remark 12. The hypothesis in Theorem 9 that $P^{\prime}$ is $S$ quasinormal in $G$ still cannot be left out when $G$ is solvable and $p$ an odd prime number.

Example 13. Let $H$ be the elementary abelian 3-group of order 27. Then $H=Z_{3} \times Z_{3} \times Z_{3}$. It is easy to see that a subgroup of $\operatorname{Aut}(H)$ is isomorphic to $Z_{13} \rtimes Z_{3}$. Now assume that $G=\left(Z_{3} \times Z_{3} \times Z_{3}\right) \rtimes\left(Z_{13} \rtimes Z_{3}\right)$. Let $P_{3}$ be a Sylow 3subgroup of $G$. It is clear that $N_{G}\left(P_{3}\right)=P_{3}$ and thus every maximal subgroup of $P_{3}$ is $S E$-supplemented in $N_{G}\left(P_{3}\right)=P_{3}$. However, $G$ is not 3-nilpotent.

Theorem 14. Let $G$ be a group, $H$ a normal subgroup of $G$ such that $G / H$ is p-nilpotent, and $P$ a Sylow p-subgroup of $H$, where $p$ is a prime dividing the order of $G$ with $(|G|, p-1)=1$. If there exists a Sylow p-subgroup $P$ of $H$ such that every maximal subgroup of $P$ is $S E$-supplemented in $N_{G}(P)$ and such that $P^{\prime}$ is $S$-quasinormal in $G$, then $G$ is p-nilpotent.

Proof. Assume that the result is not true and let $G$ with subgroup $H$ be a minimal counterexample to the theorem in respect to $|G|+|H|$. By Lemmas 2 and 4 , we can see that every maximal subgroup of $P$ is $S E$-supplemented in $N_{H}(P)$ and $P^{\prime}$ is $S$-quasinormal in $H$. It follows that $H$ is $p$ nilpotent by Theorem 9 . Let $M$ be the normal $p$-complement of $H$; then $M \unlhd G$. If $M \neq 1$, we consider the factor group $G / M$ with subgroup $H / M$. It is clear that $H=P M$ and $(|P|,|M|)=1$. With a similar argument as in step (4) of Theorem 9, we obtain that the hypothesis still holds for $G / M$ with subgroup $H / M$. Thereby the choice of $G$ implies that $G / M$ is $p$-nilpotent. Consequently, $G$ is $p$-nilpotent, which is a contradiction. Now we may suppose that $M=1$; that is, $H=P$ is a $p$-group. Let $T / P$ be the normal $p$-complement of $G / P$; this makes sense as $G / P=G / H$ is $p$-nilpotent. It is easy to see that every maximal subgroup of $P$ is $S E$-supplemented in $N_{T}(P)$ and $P^{\prime}$ is $S$-quasinormal in $T$, whence $T$ is $p$ nilpotent by Theorem 9 . As a result, $T_{p^{\prime}} \operatorname{char} T \unlhd G$ implying that $T_{p^{\prime}}$ is also a normal Hall $p^{\prime}$-subgroup of $G$; that is, $G$ is 
$p$-nilpotent, a contradiction. The proof of the theorem is now complete.

The following result now follows directly from Theorem 14.

Corollary 15. Let $G$ be a group and assume $p$ is a prime dividing the order of $G$ with $(|G|, p-1)=1$. Suppose that $H$ is a normal subgroup of $G$ such that $G / H$ is p-nilpotent. If there exists a Sylow p-subgroup $P$ of $H$ such that every maximal subgroup of $P$ is S-quasinormally embedded or weakly SEsupplemented in $N_{G}(P)$ and such that $P^{\prime}$ is $S$-quasinormal in $G$, then $G$ is p-nilpotent.

The following two theorems study the supersolvability of a group.

Theorem 16. Let $G$ be a group. Suppose that for any prime $p$ dividing $|G|$, there exists a Sylow p-subgroup $P$ of $G$ such that every maximal subgroup of $P$ is $S E$-supplemented in $N_{G}(P)$ and such that $P^{\prime}$ is $S$-quasinormal in $G$. Then $G$ is supersolvable.

Proof. Let $G$ be a minimal counterexample. According to Theorem 9, it is easy to see that $G$ is $p$-nilpotent for the minimal prime $p$ dividing $|G|$. Let $K$ be the normal $p$ complement of $G$. Then by Lemmas 2 and 4 , it is clear that $K$ meets the hypothesis of the theorem, and then $K$ is supersolvable by the choice of $G$. Let $q=\max \pi(K)$ and $Q \in \operatorname{Syl}_{q}(K)$. Then $Q \in \operatorname{Syl}_{q}(G)$ and $Q \unlhd G$. Let $N$ be a minimal normal subgroup of $G$ contained in $Q$. Thus $N$ is an elementary abelian $q$-subgroup of $G$. Now we consider the factor group $G / N$. By Lemmas 2 and 4, we know that every maximal subgroup $Q_{1} / N$ of $Q / N$ is $S E$-supplemented in $N_{G / N}(Q / N)=N_{G}(Q) / N=G / N$ and $(Q / N)^{\prime}=Q^{\prime} N / N$ is $S$-quasinormal in $G / N$. Let $R N / N \in \operatorname{Syl}_{r}(G / N)$ for any $r \neq q$. Then for any maximal subgroup $T / N$ of $R N / N$, we know that $T=R_{1} N$, where $R_{1}$ is a maximal subgroup of $R$. From the hypothesis, $R_{1}$ is $S E$-supplemented in $N_{G}(R)$. Then there is a subgroup $K$ of $N_{G}(R)$ such that $N_{G}(R)=R_{1} K$ and $R_{1} \cap K \leq\left(R_{1}\right)_{\operatorname{seN}_{G}(R)}$. Because $R \in \operatorname{Syl}_{r}(G)$, we know that

$$
N_{G / N}\left(\frac{R N}{N}\right)=\frac{N_{G}(R) N}{N}=\left(\frac{R_{1} N}{N}\right)\left(\frac{K N}{N}\right) .
$$

Since $\left(\left|R_{1}\right|,|N|\right)=1$,

$$
\begin{aligned}
\left|R_{1} \cap K N\right| & =\frac{\left(\left|R_{1}\right| \cdot|K N|_{r}\right)}{\left|R_{1} K N\right|_{r}}=\frac{\left(\left|R_{1}\right| \cdot|K|_{r}\right)}{\left|N_{G}(R) N\right|_{r}} \\
& =\frac{\left(\left|R_{1}\right| \cdot|K|_{r}\right)}{\left|N_{G}(R)\right|_{r}}=\left|R_{1} \cap K\right| .
\end{aligned}
$$

This means that $R_{1} \cap K N=R_{1} \cap K$; thus

$$
\begin{aligned}
\left(\frac{R_{1} N}{N}\right) \cap\left(\frac{K N}{N}\right) & =\frac{\left(R_{1} N \cap K N\right)}{N}=\frac{\left(R_{1} \cap K N\right) N}{N} \\
& =\frac{\left(R_{1} \cap K\right) N}{N} \leq \frac{\left(R_{1}\right)_{s e N_{G}(R)} N}{N} .
\end{aligned}
$$

Note that $\left(R_{1}\right)_{s e N_{G}(R)} N / N=\left(R_{1} N / N\right)_{s e\left(N_{G / N}(R N / N)\right)}$ by Lemma 3. Consequently, $G / N$ satisfies the hypothesis of the theorem. It follows that the choice of $G$ implies that $G / N$ is supersolvable. As the class of all supersolvable subgroups is a saturated formation, we may assume that $N$ is the unique minimal normal subgroup of $G$ contained in $Q$ with $N \not$ $\Phi(G)$, so there exists a maximal subgroup $M$ of $G$ such that $G=M N$ and $M \cap N=1$. Since $Q=O_{q}(G) \leq F(G) \leq$ $C_{G}(N), N$ and $M$ normalize $Q \cap M$, whence $Q \cap M \unlhd G$. Thus $Q \cap M=1$ or $N \leq Q \cap M$. If the latter case holds, then $N \leq M$; namely, $G=N M=M$, a contradiction. Hence $Q \cap M=1$ and $Q=N$ is a minimal normal subgroup of $G$.

Let $Q_{1}$ be a maximal subgroup of $Q$ and $T$ be a supplement of $Q_{1}$ in $G$; then $Q_{1} T=G$ and so $Q=Q \cap Q_{1} T=Q_{1}(Q \cap T)$. This means that $Q \cap T=1$. However, since $Q \cap T$ is normal in $G$ and $Q$ is a minimal normal subgroup of $G$, we have $Q \cap T=$ $Q$. Hence $T=G$ is the unique supplement of $Q_{1}$ in $G$. Then $Q_{1} \cap T \leq\left(Q_{1}\right)_{s G}$ by Lemma 6; namely, $\left(Q_{1}\right)_{s G}=Q_{1}$ is $S$ quasinormal in $G$. Thus $N_{G}\left(Q_{1}\right) \geq O^{q}(G)$ by Lemma 5 . Since $Q_{1} \triangleleft Q$, we have $Q_{1} \triangleleft Q^{q}(G)=G$. It follows from the minimal normality of $Q$ in $G$ that $Q_{1}=1$ and so $|Q|=q$. Since $G / Q$ is supersolvable, $G$ is supersolvable, a contradiction. The proof of the theorem is now complete.

Theorem 17. Let $\mathscr{F}$ be a saturated formation containing the class of all supersolvable groups $\mathcal{U}$, and assume that $G$ is a group with a normal subgroup $H$ satisfying $G / H \in \mathscr{F}$. Suppose that for any prime $p$ dividing $|H|$, there exists a Sylow $p$ subgroup $P$ of $H$ such that every maximal subgroup of $P$ is $S E$ supplemented in $N_{G}(P)$ and such that also $P^{\prime}$ is $S$-quasinormal in $G$; then $G \in \mathscr{F}$.

Proof. Suppose that the result is not true and let $G$ with subgroup $H$ be a minimal counterexample to the theorem in respect to $|G|+|H|$. By Lemmas 2 and 4 , it is clear that for any prime $p$ dividing $|H|$, there exists a Sylow $p$-subgroup $P$ of $H$ such that every maximal subgroup of $P$ is $S E$-supplemented in $N_{H}(P)$ and that $P^{\prime}$ is $S$-quasinormal in $H$. Then $H$ meets the hypothesis of Theorem 16, and thus $H$ is supersolvable. Let $q=\max \pi(H)$ and $Q \in \operatorname{Syl}_{q}(H)$; then $Q \unlhd G$. Let $N$ be a minimal normal subgroup of $G$ contained in $Q$, and consider the quotient group $G / N$. First, $(G / N) /(H / N)=G / H \in \mathscr{F}$. With a similar argument as in the proof of Theorem 16 , we can obtain that $G / N$ with subgroup $H / N$ meets the hypothesis; hence $G / N \in \mathscr{F}$ by the choice of $G$. Consequently, we may assume that $H=Q=N$ is a minimal normal subgroup of $G$. Since $\mathscr{F}$ constitutes a saturated formation, $N \not \Phi \Phi(G)$. Then there exists a maximal subgroup $M$ of $G$ such that $G=N M$ and $N \cap M=1$. Let $M_{q}$ be a Sylow $q$-subgroup of $M$. Then $G_{q}=Q M_{q}$ is a Sylow $q$-subgroup of $G$. Let $Q_{1}=Q \cap Q_{0}$, where $Q_{0}$ is a maximal subgroup of $G_{q}$ containing $M_{q}$. Then $Q_{1}$ is a maximal subgroup of $Q$ and $Q_{1} \unlhd G_{q}$. From this hypothesis, $Q_{1}$ is $S E$-supplemented in $N_{G}(Q)=G$. Let $T$ be any supplement of $Q_{1}$ in $G$; then $Q_{1} T=G$ and $Q=Q \cap Q_{1} T=$ $Q_{1}(Q \cap T)$. This means that $Q \cap T \neq 1$. We note that $Q \cap T$ is normal in $G$ and $Q$ is a minimal normal subgroup of $G$, $Q \cap T=Q$. thus $T=G$ is the unique supplement of $Q_{1}$ in $G$. As a result, we know that $Q_{1}=Q_{1} \cap T \leq\left(Q_{1}\right)_{s G}$ by 
Lemma 6. Then $\left(Q_{1}\right)_{s G}=Q_{1}$ is $S$-quasinormal in $G$. It follows that $N_{G}\left(Q_{1}\right) \geq O^{q}(G)$ by Lemma 5 . As $Q_{1} \unlhd G_{q}$, it is easy to see that $N_{G}\left(Q_{1}\right) \geq G_{q} O^{q}(G)=G$. By the minimal normality of $Q$ in $G$, it is clear that $Q_{1}=1$ and thus $Q$ is a cyclic group of order $q$. It follows that $G \in \mathscr{F}$ by [6, Lemma 2.16], a contradiction. The proof is completed.

From Theorem 17 the following corollary is immediate.

Corollary 18. Let $\mathscr{F}$ be a saturated formation containing the class of all supersolvable groups $\mathcal{U}$, and let $G$ be a group with a normal subgroup $H$ satisfying $G / H \in \mathscr{F}$. If for any prime $p$ dividing $|H|$, there exists a Sylow p-subgroup $P$ of $H$ such that every maximal subgroup of $P$ is $S$-quasinormally embedded or weakly SE-supplemented in $N_{G}(P)$ and such that also $P^{\prime}$ is $S$ quasinormal in $G$, then $G \in \mathscr{F}$.

\section{Acknowledgments}

The authors are grateful to the referee for his or her helpful report. This research was supported by NSF of China (10961007, 11161006), NSF of Guangxi (0991101, 0991102), and 2011 higher school personnel subsidy scheme of Guangxi (5070).

\section{References}

[1] D. J. S. Robinson, A Course in the Theory of Groups, Springer, New York, NY, USA, 1982.

[2] O. H. Kegel, "Sylow-Gruppen und Subnormalteiler endlicher Gruppen," Mathematische Zeitschrift, vol. 78, pp. 205-221, 1962.

[3] W. E. Deskins, "On quasinormal subgroups of finite groups," Mathematische Zeitschrift, vol. 82, pp. 125-132, 1963.

[4] P. Schmid, "Subgroups permutable [commuting] with all Sylow subgroups," Journal of Algebra, vol. 207, no. 1, pp. 285-293, 1998.

[5] A. Ballester-Bolinches and M. C. Pedraza-Aguilera, "Sufficient conditions for supersolubility of finite groups," Journal of Pure and Applied Algebra, vol. 127, no. 2, pp. 113-118, 1998.

[6] A. N. Skiba, "On weakly s-permutable subgroups of finite groups," Journal of Algebra, vol. 315, no. 1, pp. 192-209, 2007.

[7] W. Guo, K. P. Shum, and F. Xie, "Finite group with some SEsupplemented subgroups," Glasgow Mathematical Journal, vol. 321, pp. 2843-2860, 2009.

[8] Y. Li and B. Li, "On minimal weakly SE-supplemented subgroups of finite groups," Journal of Algebra and its Applications, vol. 10, no. 5, pp. 811-820, 2011.

[9] Y. Li, "On weakly s-supplemented maximal subgroups of Sylow subgroups of finite groups," Advances in Mathematics, vol. 40, no. 4, pp. 413-420, 2011.

[10] W. Guo, A. N. Skiba, and N. Yang, "SE-supplemented subgroups of finite groups," Rendiconti del Seminario Matematico della Università di Padova, vol. 129, pp. 245-263, 2013.

[11] C. Li, "A note on a result of Skiba," Journal of Group Theory, vol. 15, no. 3, pp. 385-396, 2012.

[12] P. Hall, "On a Theorem of Frobenius," Proceedings of the London Mathematical Society, vol. 40, no. 1, pp. 468-501, 1936.

[13] S. Li and Y. Li, "On S-quasinormal and c-normal subgroups of a finite group," Czechoslovak Mathematical Journal, vol. 58, no. 4, pp. 1083-1095, 2008.
[14] Y. M. Li, Y. M. Wang, and H. Q. Wei, "On p-nilpotency of finite groups with some subgroups $\pi$-quasinormally embedded," Acta Mathematica Hungarica, vol. 108, no. 4, pp. 283-298, 2005.

[15] W. Guo and A. N. Skiba, "Finite groups with given $s$-embedded and $n$-embedded subgroups," Journal of Algebra, vol. 22, pp. 287-290, 1998.

[16] B. Huppert, Endliche Gruppen. I, Springer, Berlin, Germany, 1967. 


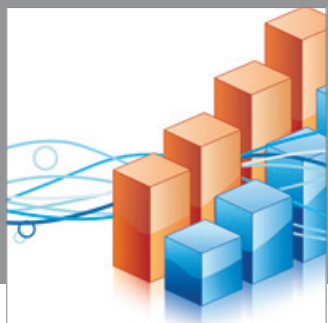

Advances in

Operations Research

mansans

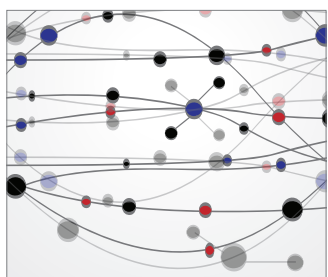

The Scientific World Journal
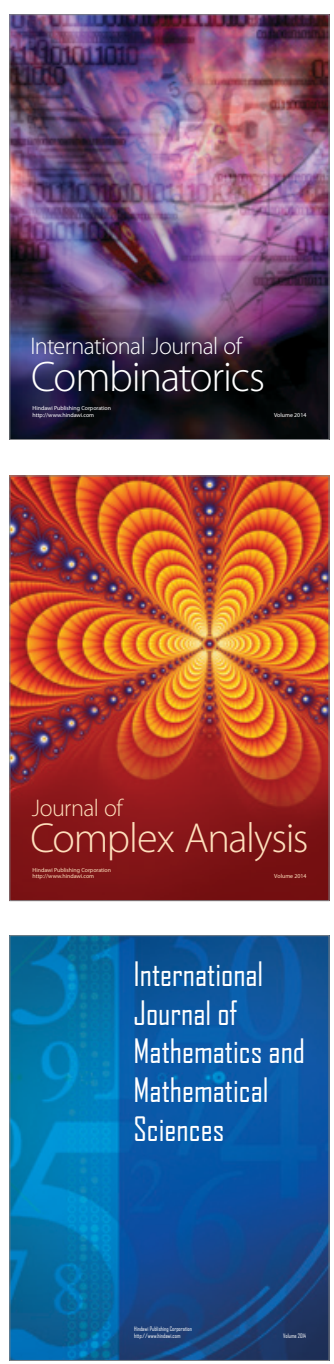
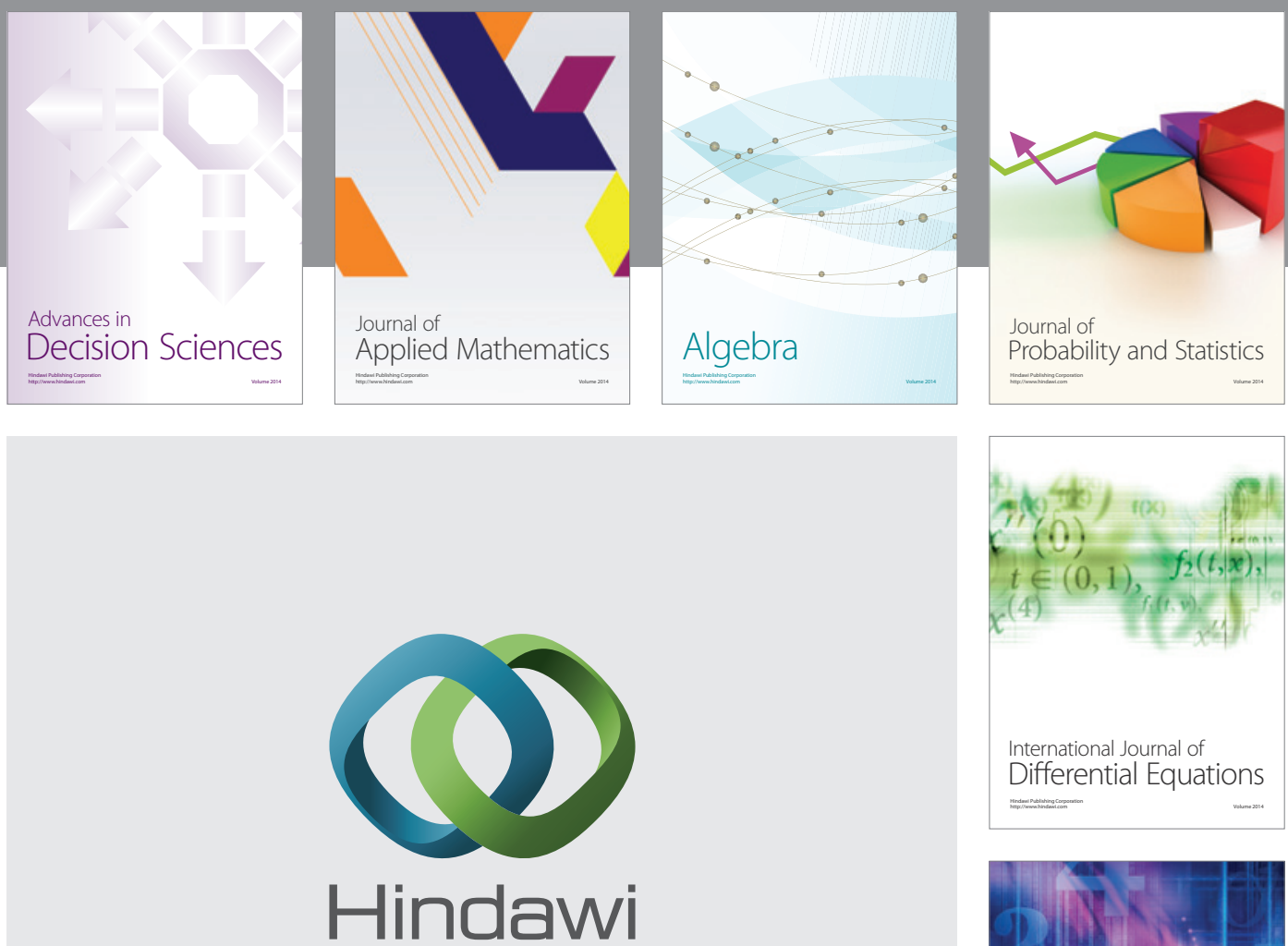

Submit your manuscripts at http://www.hindawi.com
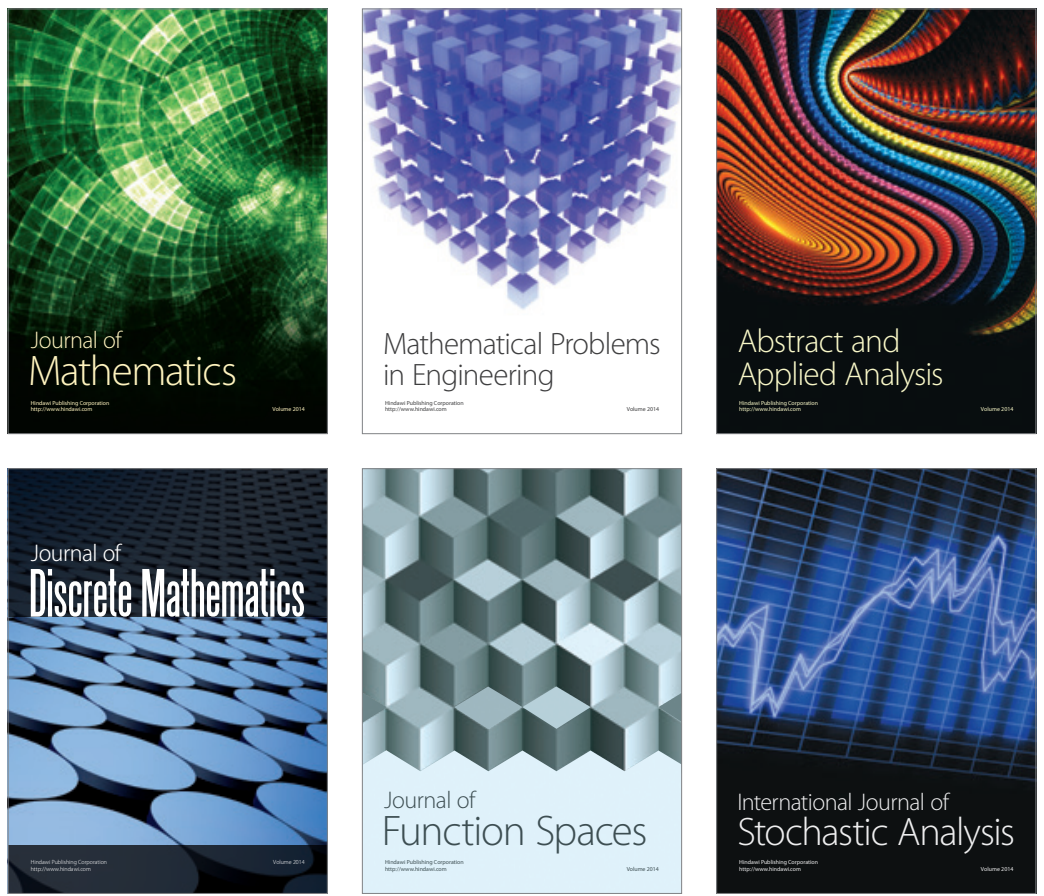

Journal of

Function Spaces

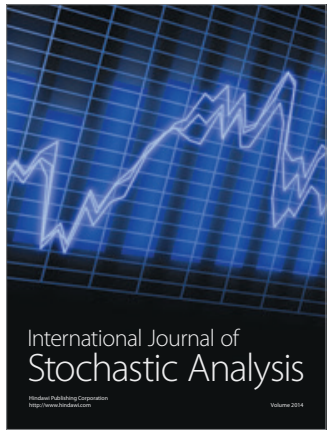

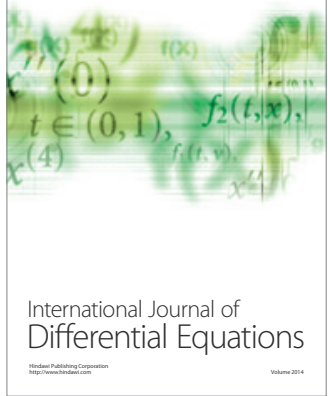
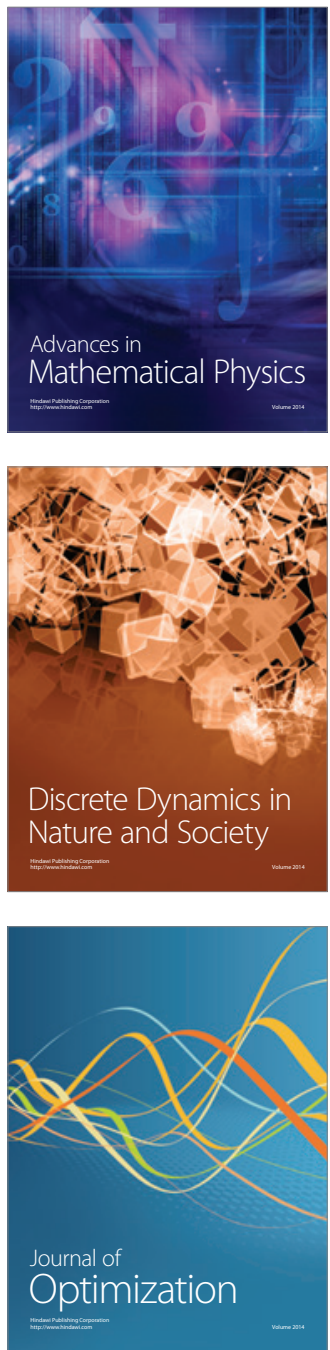